\title{
Universal CubeSat Platform Design Technique
}

\author{
Zhiyong Chen ${ }^{1, a}$ \\ ${ }^{1}$ Interligent Manufacturing Key Laboratory of Ministry of Education, Shantou University, Shantou, China
}

\begin{abstract}
This article summarizes cubesat technology, provides examples of their scientific impact, and describes the design and the manufacturing of a Cubesat platform. As for the design of the overall frame structure of the CubeSat, we have searched a lot of literature and consulted many predecessors' designs, and collected many satellite structure images. After analyzing the data, we aimed at all kinds of different structures' advantages and disadvantages, finally we got a best design.

It is a satellite of cubic shape $(10 \mathrm{~cm}$ per side), weighing approximately $1 \mathrm{~kg}$, based on the creation of a central body made of different material using the 3D-Printing techniques. The 3D-Printing technique has several advantages including fast implementation, accuracy in manufacturing small parts and low cost. Moreover, concerning the construction of a small satellite, this technique is very useful thanks to the accuracy achievable in details, which are sometimes difficult and expensive to realize with the use of tools machine.

The structure must be able to withstand the launch loads. For this reason, several simulations using an FEM simulation and an intensive vibration test campaign will be performed in the system development and test phase.
\end{abstract}

\section{Introduction}

\subsection{CubeSats History}

CubeSats are a class of nanosatellites that use a standard size and form factor. The standard CubeSat size uses a "one unit" or "1U" measuring $10 \times 10 \times 10 \mathrm{~cm}$ and is extendable to larger sizes: 1.5, 2, 3, 6, and even 12U (Figure 1). Originally developed in 1999 by California Polytechnic State University at San Luis Obispo (Cal Poly) and Stanford University to provide a platform for education and space exploration. The development of CubeSats has advanced into its own industry with government, industry and academia collaborating for ever increasing capabilities. CubeSats now provide a cost effective platform for science investigations, new technology demonstrations and advanced mission concepts using constellations, swarms disaggregated systems.

a Corresponding author : 17zychen5@stu.edu.cn 


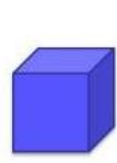

1U

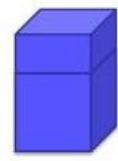

$1.5 \mathrm{U}$

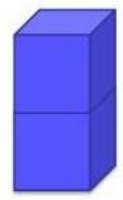

$2 \mathrm{U}$

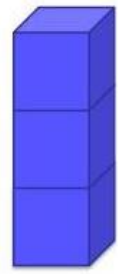

$3 \mathrm{U}$

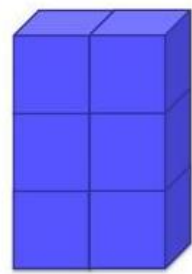

6U

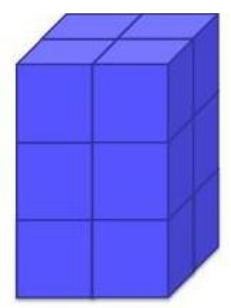

$12 \mathrm{U}$

Figure 1. The standard CubeSat size uses a "one unit" or "1U" [1]

NASA Ames launched its first CubeSat, GeneSat, in December 2006. Since then Ames has launched 16 CubeSat spacecraft varying in size from $1 \mathrm{U}$ to $3 \mathrm{U}$ with an additional 12 CubeSats in development or awaiting launch. In 2014, two 6U Perseus-M CubeSats were launched for maritime surveillance, those two CubeSats represent the largest CubeSats flown as of 2015. The 2018 launch of the InSight lander to Mars, will include two 6U CubeSats called Mars Cube One (MarCO) [2-4].

NASA has selected 34 small satellites from 19 states and the District of Columbia to fly as auxiliary payloads aboard missions planned to launch in 2018, 2019 and 2020. Launch opportunities are leveraged from existing launch services for government payloads as well as via dedicated CubeSat launches from the new Venture Class Launch Services contracts. The proposed CubeSats come from educational institutions, universities, non-profit organizations and NASA centers [5].

This article summarizes CubeSat technology, provides examples of their scientific impact, and describes the design and the manufacturing of a CubeSat platform.

\section{Main tasks}

\subsection{CubeSat Design}

The CubeSat specification accomplishes several high-level goals. The main reason for miniaturizing satellites is to reduce the cost of deployment and are often suitable for launch in multiples, using the excess capacity of larger launch vehicles. The CubeSat design specifically minimizes the risk to the launch vehicle and payloads. Encapsulation of the launcher-payload interface takes away the amount of work that would previously be required for mating a piggyback satellite with its launcher [6].

Standard CubeSats are made up of $10 \times 10 \times 11.35 \mathrm{~cm}$ units designed to provide $10 \times 10 \times 10 \mathrm{~cm}$ or 1 liter of useful volume while weighing no more than $1.33 \mathrm{~kg}(2.9 \mathrm{lb})$ per unit. The smallest standard size is $1 \mathrm{U}$ [7]. Some Aerospace Corporation has constructed and launched two smaller form CubeSats of $0.5 \mathrm{U}$ for radiation measurement and technological demonstration [8]. In recent years larger CubeSat platforms have been proposed, most commonly $6 \mathrm{U}(10 \times 20 \times 30 \mathrm{~cm}$ or $12 \times 24 \times 36 \mathrm{~cm})$ and $12 \mathrm{U}(20 \times 20 \times 30 \mathrm{~cm}$ or $24 \times 24 \times 36 \mathrm{~cm}$, to extend the capabilities of CubeSats beyond academic and technology validation applications and into more complex science and national defense goals [9].

\subsection{CubeSat Structure}

As for the design of the overall frame structure of the CubeSat, we have searched a lot of literature and consulted many predecessors' designs, and collected many satellite structure images as shown below (Figure 2): 


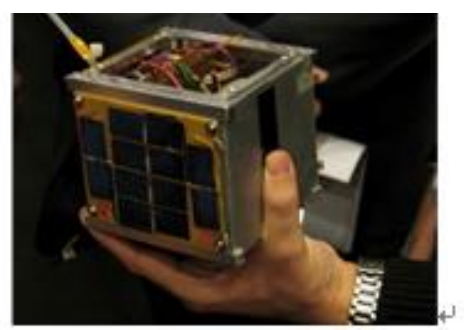

(a)

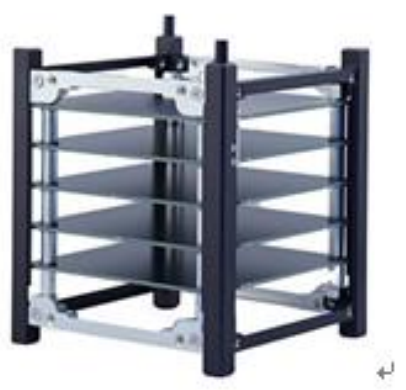

(c)

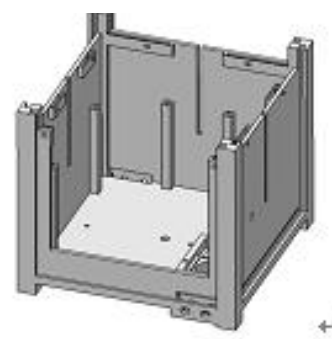

(b)

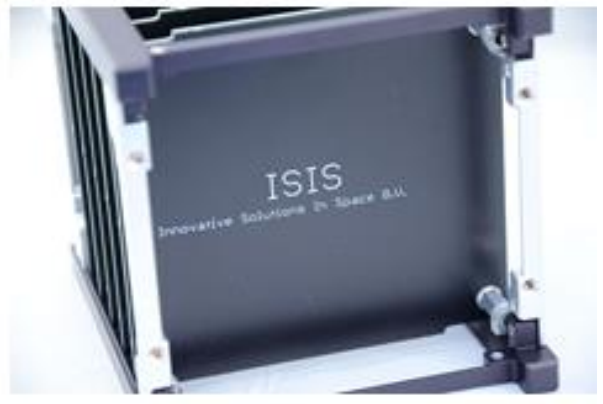

(d)

Figure 2. Some collected CubeSat structures [10-12].

In figure 2, the part (a) shows a relatively old cube model [10], the metal shell structure as a whole, contain the necessary collector and circuit board, it is the total weight of not more than $1 \mathrm{~kg}$, but the metal shell weight is large, which means it cannot carry a large amount of load. Obviously, we want a bigger payload. And part (b) shows us a clear high-level design [11], the structure of the side panels can be separated from the base, which means that we can more easily placed the satellite payload, and because of the side panel and the base is not whole, we can use different materials to meet the demand of intensity. Part (c) and (d) are two pictures of the same cube [12]. This is a structure without the side panel, and using the lightweight composite materials, this means that the structure can guarantee the quality of strength requirement and reduce it, so we can carry more load effectively.

In order for this satellite structure to be universal, in other words, to be able to adapt to various carrier rocket connections, we designed specific joints for the structure. Meanwhile, we have specifically considered how to achieve larger payload volume while reducing the structure's mass.

Despite its small size, this single unit (1U) CubeSat should be equipped with several necessary subsystems, an active attitude control, a developed telecommunication system and high efficiency solar cells for power supply. The subsystems developed for this CubeSat have also been designed to be scaled up for larger satellites such as $2 \mathrm{U}$ or $3 \mathrm{U}$ CubeSats. The additional volume can be used for more payloads. So that the satellite can be used as a low cost platform for companies, institutions or universities to test components in space. 

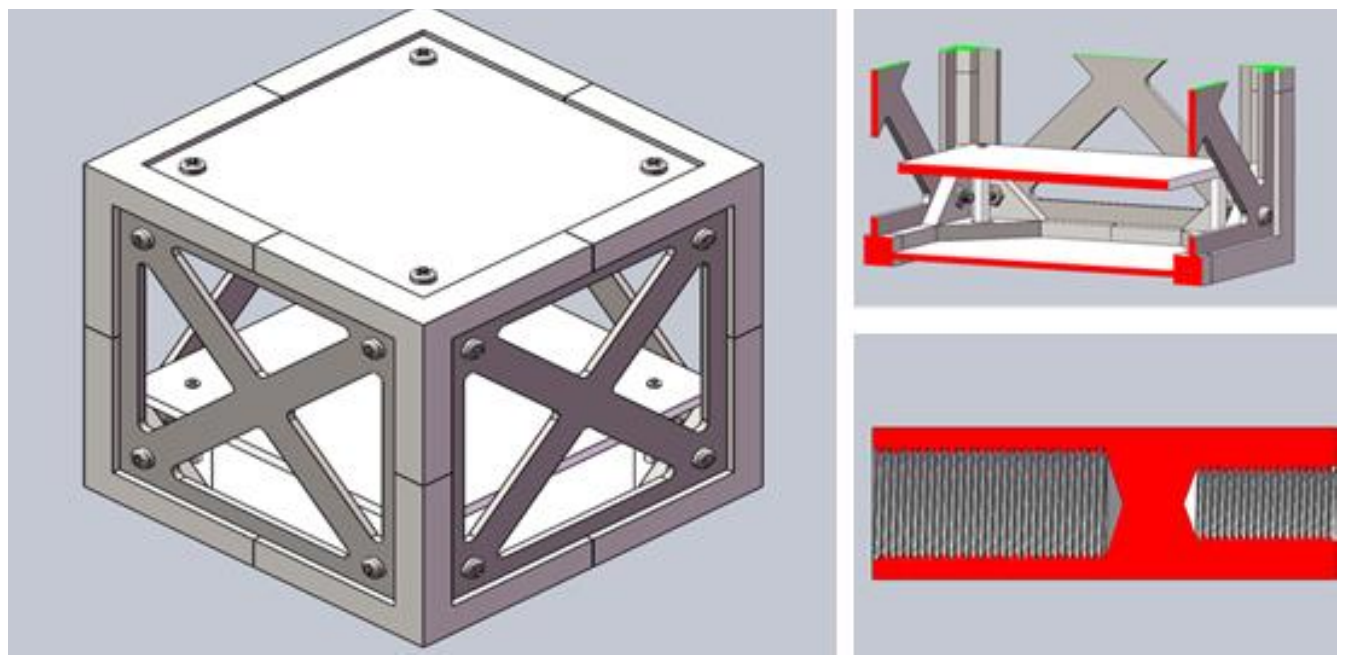

Figure 3. Completed structure design (left) \& Structural design details \& the plate support column of the electronic component (right).

After summarizing all the structures investigated, we have come up with the following structure design (Figure 3). As shown in the figure 3, the whole structure can be decomposed into two of the same top and bottom plates, four equal sides and eight exactly the same bases. It makes the manufacturing process of the structure simplified.

Such a design also facilitates the installation and disassembly of the whole structure. At the same time, we make the interior of the structure as large as possible to carry the payload. The design of the side plate is to reduce the mass under the premise of ensuring the strength of the structure. There are more details about the design structure (Figure 3).

\section{Manufacturing technology}

The CubeSat structure can be made of different material using the 3D-Printing techniques, for example, plastic materials, aluminum, gray cast iron, copper or other composite materials and so on it depends on our different design requirements (structural strength, mass requirements, etc.) for the structure. The 3D-Printing technique has several advantages including fast implementation, accuracy in manufacturing small parts and low cost. Moreover, concerning the construction of a small satellite, this technique is very useful thanks to the accuracy achievable in details, which are sometimes difficult and expensive to realize with the use of tools machine.

The advantage of the manufacturing technology is that it can produce product with very complex shape, and these products are impossible to implement in the form of low cost, or made in a short period with traditional manufacturing methods. In addition, this technique has changed the concept of general and secondary structures. Usually, the traditional manufacturing technology is aimed to remove material from the metal base, while 3D-printing, on the contrary, is covering the material layer by layer, so the parts are produced at the same time, the product is a whole. It reduces the number of parts, also reduces the screws and the needs of the adhesive, and simplifies the assembly process, at the same time, the reliability of the structure improved [11].

\section{Simulations}

The structure must be able to withstand the launch loads. For this reason, several simulations using an FEM simulation and an intensive vibration test campaign will be performed in the system development and test phase [13]. In addition, according to the requirements of the orbital environment, 
the most critical environmental tests and the critical components selection will be carried out.

Environmental testing, including the architecture of satellite vacuum test and thermal cycling test. Before and after the test, we hope that the structure mass won't be changed obviously, and during the pure cycle simulation of low earth orbit conditions (about an orbital period, temperature changes from $-20^{\circ} \mathrm{C}$ to $80^{\circ} \mathrm{C}$ ), the quality of the satellite architecture will change no more than $1 \%$. Moreover, a thermal shock test of structure should be taken, in order to response to sudden temperature changes (temperature range from $-20^{\circ} \mathrm{C}$ to $60{ }^{\circ} \mathrm{C}$ ).

The effects of radiation on satellite structures are thought to be negligible. According to the results of Ref. [14], in order to has serious damage to the structure, the total dose of radiation needs to be up to $10^{9} \mathrm{rad}(\mathrm{Si})$, however, any LEO orbit satellite 1 year experiment operating radiation (receiving less than $10^{7} \mathrm{rad}(\mathrm{Si}), 2000 \mathrm{~km}$ height [15]) is far lower than this value. In addition, with regard to direct solar radiation, it is necessary to consider that the structure almost completely covers the solar cells, while solar cells block the main parts of the structure.

\section{Conclusion}

CubeSats are playing an increasingly larger role in exploration, technology demonstrations, scientific research and education at university. These miniature satellites provide a low-cost platform for national research missions, including planetary space exploration; Earth observation; fundamental Earth and space science; and technology demonstrations such as cutting-edge laser communications, energy storage, in-space propulsion and autonomous movement capabilities. They also provide educators an inexpensive means to engage students in all phases of satellite development, operation and exploitation through real world; hands-on research and development experience on NASA-funded ride-share launch opportunities.

A 1 U Cubesat system has been developed aimed at education, low cost realization and research. The system includes innovative solutions, potentially improving the overall performance, flexibility and cost. Ground test results show that these are all feasible and promising. Under using structural and parametric conception to design universal multipurpose satellite platform CubeSat and improve modern technological process (3D printing and additive manufacturing) during it manufacture.

\section{References}

1. Elizabeth, What are SmallSats and CubeSats? (Mabrouk, Feb. 26, 2015).

2. MarCO, Planetary CubeSats Become Real. (www.planetary.org, Retrieved 2016-02-23).

3. Clark, Stephen. Launch of NASA's next Mars mission delayed until at least 2018. (Spaceflight Now, Retrieved 2016-02-23).

4. "CubeSat". Space.Skyrocket.de. Retrieved 2015-10-18.

5. NASA Announces Eighth Class of Candidates for Launch of CubeSat Space Missions, Editor: Shanessa Jackson, Feb. 21, 2017.

6. CubeSat - https://en.wikipedia.org/wiki/.

7. Mehrparvar, Arash, "CubeSat Design Specification" (PDF). (February 20, 2014).

8. AeroCube 6A, 6B (CubeRad A,B)., www.space.skyrocket.de, Retrieved 2015-10-18.

9. The CubeSat Program, CalPoly SLO. Retrieved March 25, 2017.

10. Kirk Woellert, Pascale Ehrenfreund, Antonio J. Ricco, Henry Hertzfeld. Cubesats: Cost-effective science and technology platforms for emerging and developing nations. Applied ScienceDirect. 47(2011): 663

11. Jacopo Piattoni, Gian Paolo Candini, Giulio Pezzi, Fabio Santoni, Fabrizio Piergentili. Plastic Cubesat: An innovative and low-cost way to perform applied space research and hands-on education. Applied Elsevier. 81(2012): 420

12. One-stop webshop for CubeSats and Nanosats - https://www.cubesatshop.com/. 
13. Athirah, Nur; Afendi, Mohd; Hafizan, Ku; Amin, N.A.M.; Majid, M.S. Abdul. "Stress and Thermal Analysis of CubeSat Structure". Applied Mechanics and Materials. 554: 426-430. Doi: 10.4028/www.scientific.net/amm.554.426.

14. NASA Space Vehicle Design Criteria (Structure), NASA SP-8053, June 1970.

15. W.J. Larson, J.R. Wertz, Space Mission Analysis and Design, third edition, Space Technology Library-Microcosm Press, California, 2004. 